\title{
Role of Apo E and superoxide dismutase in patients with obstructive lung diseases
}

\author{
Anwar J Almzaiel, ${ }^{a}$ Ali Mansoor Jasim Al-Ameri, ${ }^{\text {b Rafad Tariq }}{ }^{c}$
}

\author{
'Department of biochemistry, College of Medicine, University of Alqadisiyah, Iraq. \\ ${ }^{b}$ Department of Microbiology and Immunology College of Medicine, University of Kerbala,Kerbala, Iraq. \\ ${ }^{b}$ Master Biochemistry student, Department of biochemistry, College of Medicine, University of Kerbala, Kerbala, Iraq. \\ Correspondence to: anwar.almzaiel@qu.edu.iq. \\ (Submitted: 09 December 2017 - Revised version received: 11 January 2017 - Accepted: 16 February 2017 - Published online: 26 March 2017)
}

\begin{abstract}
Objective Obstructive lung diseases (OLD) are chronic inflammatory disorders of the respiratory tract including asthma and chronic obstructive pulmonary disease (COPD). Apo lipoprotein E (Apo E), is a multifunctional protein as it intervenes the binding of lipoproteins or lipid complexes to specific cell-surface receptors. Experimental studies referred to the function of Apo $E$ as an endogenous negative regulator of airway hyper responsiveness and goblet cell hyperplasia. The protective role of Apo E pathways primarily in respiratory disease was explained in human studies and research utilizing experimental murine model systems. Literature data reveal a strong association between redox status, including the enzyme superoxide dismutase (SOD) with both the development a severity of OLD. This study aims to investigate the relation between SOD antioxidant enzyme activity in addition to investigating the level of Apo E and the development of obstructive lung diseases (OLD).

Methods Patients with OLD $(n=40)$ and 40 age-matched healthy controls were enrolled in this study. Serum samples were collected to test the role of Apo E and to test the effect of antioxidant enzyme SOD, and their influence on OLD, all measured by ELISA.

Result The results showed a significant decrease in the level of serum SOD activity in patients with OLD when compared with control group $(P<0.05)$. However, the levels of Apo E did not show a significant difference between the two groups.

Conclusion Decreased level of antioxidant SOD suggests the presence of an oxidative stress in asthmatic airways favoring a more oxidative state is present in the airway inflammation.

The level of Apo E was non significantly increased in serum of patient, this suggests that protein level of Apo E does not change but may be Apo E gene expression is altered.

Keywords apo E, superoxide dismutase, obstructive lung diseases
\end{abstract}

\section{Introduction}

Chronic respiratory diseases such as asthma and chronic obstructive pulmonary disease (COPD) are common problems and are regarded as the major public health burdens. ${ }^{1,2}$ They are characterized by reversible and irreversible airway obstruction, respectively. Although remain the primary reason of these diseases unknown, progressive and permanent pulmonary tissue damage (airway remodeling) that leads to the total loss of lung function as a result of inflammation which is considered a central feature for them. In fact, the inflammatory mechanisms and other biological pathways involved in asthma and COPD pathogenesis must be explained, in order to find new possible diagnostic/prognostic biomarkers and for the validation of new drug targets. ${ }^{3}$

There are significant differences in the patterns of fundamental inflammation, in spite of the similarity in clinical symptoms of both diseases, they are caused by airway narrowing as a result of inflammation.

In COPD, the inflammatory cell infiltrate in small airways include mainly neutrophils and cytotoxic T cells (cluster of differentiation-8 (CD8) positive lymphocytes). Parenchymal destruction in COPD is associated with loss of lung tissue elasticity, and small airways collapse during exhalation. Asthma has been characterized mainly by Type 2 helper T cell (Th2) cytokine-mediated eosinophilic airway inflammation associated with airway hyperresponsiveness.

Recent studies suggest that $13 \%-20 \%$ of patients with COPD have an overlap phenotype with asthma; this is as high as $50 \%$ in patients over 50 years, because of $30 \%$ of people with asthma smoke, a proportion of these will develop chronic airflow limitation, which is likely to be indistinguishable from COPD. ${ }^{5-7}$

Antioxidants are one of chemical substances can inhibit the oxidation of a molecule. In the living organisms, antioxidants can nullify the pathology effects of oxidation caused by free radicals.

\section{Superoxide Dismutase}

Superoxide dismutase is considered one of oxidoreductases , which catalyze the dismutation of $\mathrm{O}_{2}^{-{ }^{-}}$into oxygen and $\mathrm{H}_{2} \mathrm{O}_{2}$, therefor it is classified as a major cellular defense against $\mathrm{O}_{2}{ }^{-}$ and peroxynitrite.

Thus, $\mathrm{Cu}$ or $\mathrm{Mn}$ will be important modulator of SOD activity of SOD1/SOD3 or SOD2, respectively.

\section{Apo Lipoproteine $E$}

Apo lipoproteine E (Apo E) is a protein presents in the interstitial fluid and lymph, as well as in the plasma and is synthesized and secreted from a variety of tissues and different kinds of cells. ${ }^{10-12}$ It has three major isoforms (Apo E2, Apo E3, and Apo E4) with different effects on lipid and neuronal homeostasis. ${ }^{13}$

Intracellular Apo E may regulate various cellular processes physiologically or pathophysiologically. Apo lipoprotein (Apo) $\mathrm{E}$ is a multifunctional protein as it intervenes the binding of lipoproteins or lipid complexes in the plasma or interstitial fluids to specific cell-surface receptors as a major function of Apo E. ${ }^{13}$ 
Many respiratory diseases, including asthma, acute lung injury, cancer, emphysema, pulmonary fibrosis, and pulmonary hypertension are associated with Apo $\mathrm{E}$ which is expressed by lung cells, which allows Apo E/ low density lipoprotein receptor (LDLR) dependent pathways to modulate normal lung health, as well as the pathogenesis of these diseases. The protective roles of Apo E and Apo lipoproteine A-I (Apo A-I) pathways primarily in lung biology and respiratory disease were explained in human studies and research utilizing experimental murine model systems. ${ }^{14}$

\section{Materials and Methods}

\section{Assay Procedure}

Serum levels of superoxide dismutase were determined by classic Competitive-ELISA using ELISA minikits (Elabscience, China). Whereas serum levels of Apo E were determined by classic Sandwich-ELISA using ELISA minikits (Elabscience, China) according to the instructions enclosed with the kits.

\section{Calculation of Results}

Average the duplicate readings for each standard and sample. Create a standard curve by plotting the mean OD value for each standard on the $y$-axis against the concentration on the $\mathrm{x}$-axis and draw a best-fit curve through the points on the graph. By using professional Microsoft Excel to do this calculation, a best fitting equation of standard curve will be calculated using OD values and concentrations of standard sample. The software will calculate the concentration of samples after entering the OD value of samples.

\section{Results}

The antioxidant status which represented in the present study was reduced SOD which is the master antioxidant in the body. This was achieved by evaluating the changes according to the activity of the disease. A brief illustration in (Table 1) which summarize the overall findings regarding the studied parameters of the current study.

\section{Superoxide dismutase}

The results in Fig. 1 show a significant decrease $(P<0.05)$ in SOD concentration in serum of patient with OLD group compared to the control group.

The data show that the mean \pm SD of SOD in patients with OLD and control group were $445.702 \pm 148.217$ and $520.757 \pm$ 179.902 Pg/ml, respectively.

\begin{tabular}{llcc}
\hline Table 1. & \multicolumn{3}{c}{$\begin{array}{c}\text { Summarizes parameters studied represented by serum } \\
\text { (SOD and Apo E) for control and patients with OLD }\end{array}$} \\
\hline Parameters & Group & Mean \pm SD & P-value \\
\hline \multirow{2}{*}{ SOD; $\mathrm{pg} / \mathrm{ml}$} & Patient & $445.702 \pm 148.217$ & $<0.05$ \\
& Control & $520.757 \pm 179.902$ & \\
& Patient & $727.47 \pm 189.31$ & $>0.05$ \\
Apo E; $\mathrm{ng} / \mathrm{ml}$ & Control & $697.51 \pm 207.32$ & \\
&
\end{tabular}

SOD, superoxide dismutase; Apo E, apo lipoprotein E; OLD, obstructive lung disease; Student's $t$-test $P$-value of $<0.05$ was considered to be statistically significant.

\section{Apo Lipoproteine E}

The results in Fig. 2 showed non-significant change $(P>0.05)$ in serum Apo E concentration in patients with OLD group compared to the control group. The data show that the mean \pm SD of Apo E in OLD and control group were 727.47 \pm 189.31 and $697.51 \pm 207.32 \mathrm{Pg} / \mathrm{ml}$, respectively.

\section{Discussion}

The results of the present study revealed a significant decrease in SOD levels in patients with OLD. An enzymatic antioxidant system is critical for the redox status homeostasis and for protecting human cells. In fact, when this homeostasis is disrupted and there are more reactive species than antioxidants several diseases can develop. ${ }^{1}$

Recent studies indicate that an increased oxidative stress is related to the pathogenesis of COPD, ${ }^{1,16-18}$ and contribute this to the fact that COPD patients produce more $\mathrm{H}_{2} \mathrm{O}_{2}$ compared to "healthy". ${ }^{19}$ Another study hypothesized that loss of asthmatic SOD activity is due to greater susceptibility to oxidative inactivation. ${ }^{20}$

An increase in ROS generation in asthma and/or COPD patients is confirmed by the changes in the antioxidant

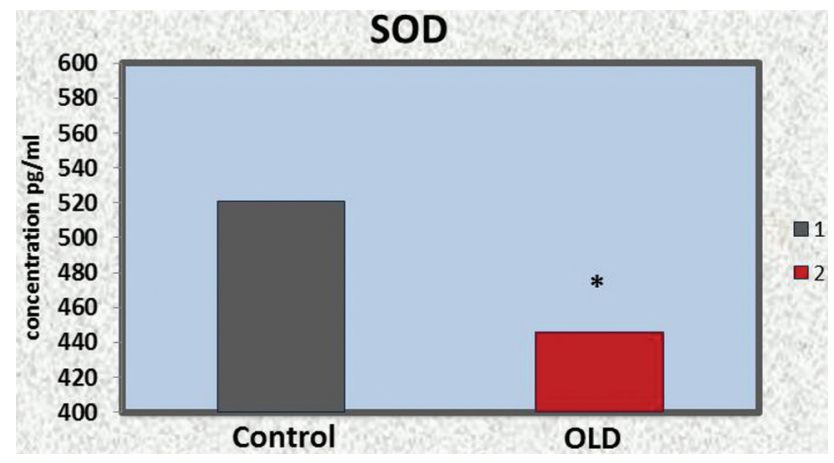

Fig. 1 Serum superoxide dismutase (SOD) activity in patients with obstructive lung disease (OLD) and control. Serum samples were isolated from the blood of patients with OLD. SOD level was assessed by ELISA. Data are expressed as means \pm SD, for 40 patients, with duplicate measurements. *indicates significant differences compared to the control $n=40$, (Student's $t$-test, $P<0.05$ ).

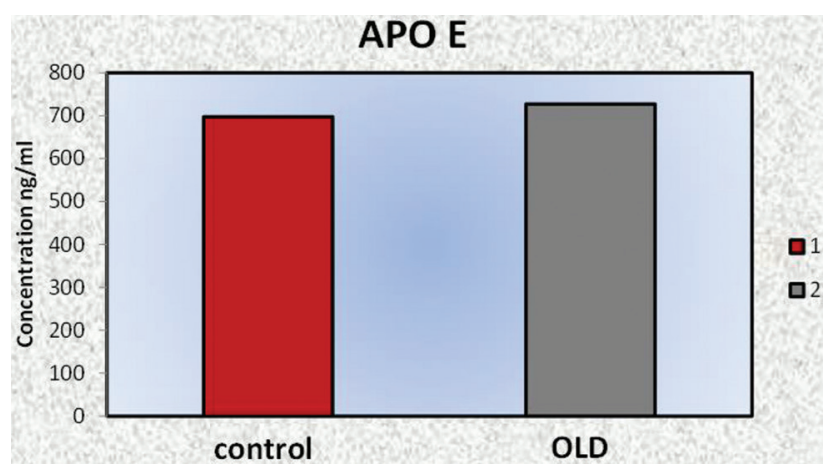

Fig. 2 Serum apo lipoprotein E (Apo E) levels in patients with obstructive lung disease (OLD) and control. Serum samples were isolated from the blood of patients with OLD. Apo E level was assessed by ELISA. Data are expressed as means \pm SD, for 40 patients, with duplicate measurements, compared to the control group $n=40$, (Student's $t$-test, $P>0.05$ ). 
enzymes activity. These results support the hypothesis that an oxidant-antioxidant imbalance, associated with oxidative stress in COPD patients, plays an important role in the progression of disease severity. ${ }^{21}$

Previous studies also observed the same fact and concluded that oxidative stress remains increased among asthmatics with low levels of antioxidant activity. ${ }^{22}$

There is a strong evidence that an imbalance between the reducing and oxidizing systems favoring a more oxidative state is present in the airway inflammation and a deficiency in the amount of antioxidants exists in the asthmatic airway. ${ }^{23}$

Superoxide dismutases (SODs) are the major antioxidant defense systems against $\mathrm{O}_{2}$ radical. ${ }^{24}$

Vulnerability of CuZnSOD influenced by redox likely amplifies injury and inflammation during acute asthma attacks when reactive oxygen species are explosively generated. Overall, this study identifies a new paradigm for understanding the chemical basis of inflammation..$^{20}$

The difference in mean SOD level was found to significantly decrease among asthmatic patients. ${ }^{25}$

Antioxidants not only protect against the direct injurious effects of oxidants, but also alter the inflammatory events that play an important role in the pathogenesis of COPD. ${ }^{26}$ The outcome of the present investigation confirms the role of an oxidant-antioxidant imbalance in the etiology of COPD and/or asthma. The data from the current study have shown non-significant increase in the Apo E levels in patients when compared with control group (Fig. 2). This finding is consistent with the results of many previous studies as shown below.

Although the primary function of Apo E is to facilitate lipid transport into cells by receptor-mediated endocytosis mediated by the LDL receptor, it is has been recognized that Apo E modulates a variety of additional important biological functions. Recent studies suggested that Apo E may be involved in therapy strategy for OLD.

Experiments utilizing humanized Apo E knock-in mice have demonstrated that human Apo E\&3 had a protective effect on the severity of HDM-induced airway disease. ${ }^{27}$

The ability of Apo E, which is expressed by lung macrophages, to attenuate AHR, and goblet cell hyperplasia is mediated by low density lipoprotein (LDL)receptors expressed by airway epithelial cells. So, the administration of an Apo E mimetic peptide, corresponding to amino acids 130-149 of the LDL receptor-binding domain of the holo-Apo E protein, significantly reduced AHR and goblet cell hyperplasia in HDM-challenged Apo E-/-mice.

These findings identified the Apo E-LDL receptor pathway as a new drug able target for asthma that can be activated by administration of Apo E-mimetic peptides..$^{28}$

\section{Recommendation}

Further studies are to be conducted to predict the causal association of other markers such as lipid profile, Apo A and non-enzymatic antioxidants with the development of OLD; asthma and COPD.

It is worthy to investigate Apo $\mathrm{E}$ in cell culture and study the different effect of polymorphic Apo E alleles by using gene expression.

\section{Conflicts of interest}

None.

\section{References}

1. Lundgren-Nilsson $\AA$, Jonsdottir IH, Pallant J, Ahlborg G. Internal construct validity of the Shirom-Melamed Burnout Questionnaire (SMBQ). BMC Public Health. 2012;12:1.

2. Abramson MJ, Perret JL, Dharmage SC, McDonald VM, McDonald CF. Distinguishing adult-onset asthma from COPD: a review and a new approach. Int J Chron Obstruct Pulmon Dis. 2014;9:945-962.

3. Rossi R, De Palma A, Benazzi L, Riccio AM, Canonica GW, Mauri P. Biomarker discovery in asthma and COPD by proteomic approaches. PROTEOMICSClinical Applications. 2014:8:901-915.

4. Barnes PJ. Immunology of asthma and chronic obstructive pulmonary disease. Nat Rev Immunol, 2008a;8:183-192.

5. Thomson NC, Chaudhuri R, Livingston E. Asthma and cigarette smoking. Eur Respir J. 2004;24:822-833

6. Broekema M, Timens W, Vonk JM, Volbeda F, Lodewijk ME, Hylkema MN, et al. Persisting remodeling and less airway wall eosinophil activation in complete remission of asthma. Am Journal of respiratory and critical care medicine. 2011;183:310-316.

7. Piras B, Miravitlles M. The overlap phenotype: the (missing) link between asthma and COPD. Multidisciplinary respiratory medicine. 2012;7:1.

8. Singh PP, Chandra A, Mahdi F, Roy A, Sharma P. Reconvene and reconnect the antioxidant hypothesis in human health and disease. Indian J Clin Biochem. 2010;25:225-243.

9. Abreu IA, Cabelli DE. Superoxide dismutases - a review of the metalassociated mechanistic variations. Biochimica et Biophysica Acta (BBA)Proteins and Proteomics. 2010;1804:263-274.

10. Huang Y. Abeta-independent roles of apolipoprotein E4 in the pathogenesis of Alzheimer's disease. Trends Mol Med. 2010;16:287-294.

11. Huang Y, Mucke L. Alzheimer mechanisms and therapeutic strategies. Cell. 2012;148:1204-1222.

12. Mahley RW, Huang Y. Apolipoprotein e sets the stage: response to injury triggers neuropathology. Neuron. 2012;76:871-885.

13. Huang Y, Mahley RW. Apolipoprotein E. structure and function in lipid metabolism, neurobiology, and Alzheimer's diseases. Neurobiol Dis. 2014;3-12.

14. Yao X, Gordon EM, Figueroa DM, Barochia AV, Levine SJ. Emerging Roles of Apo lipoprotein E and Apo lipoprotein Al in the Pathogenesis and Treatment of Lung Disease. Am J Respir Cell Mol Biol. 2016;55:159-169.

15. Velpandian T, Gupta P, Ravi AK, Sharma HP, Biswas NR. Evaluation of pharmacological activities and assessment of intraocular penetration of an ayurvedic polyherbal eye drop (Itone ${ }^{\mathrm{TM}}$ ) in experimental models. BMC Complement Altern Med. 2013;13:1

16. Cristovao C, Cristóvão L, Nogueira F, Bicho M. Evaluation of the oxidant and antioxidant balance in the pathogenesis of chronic obstructive pulmonary disease. Revista Portuguesa de Pneumologia (English Edition). 2013;19:70-75.

17. Barreiro E, Fermoselle C, Mateu-Jimenez M, Sánchez-Font A, Pijuan L, Gea J, et al. Oxidative stress and inflammation in the normal airways and blood of patients with lung cancer and COPD. Free Radic Biol Med. 2013;65:859-871.

18. Domej W, Oettl K, Renner W: Oxidative stress and free radicals in COPDimplications and relevance for treatment. Int J Chron Obstruct Pulmon Dis 2014;9:1207-1224.

19. Möller W, Heimbeck I, Weber N, Khadem Saba G, Körner B, Neiswirth M, Kohlhäufl M. Fractionated exhaled breath condensate collection shows high hydrogen peroxide release in the airways. J Aerosol Med Pulm Drug Deliv. 2010;23:129-135.

20. Ghosh S, Willard B, Comhair SA, Dibello P, Xu W, Shiva S, et al. Disulfide bond as a switch for copper-zinc superoxide dismutase activity in asthma. Antioxid Redox Signal 2013;18:412-423.

21. Ahmad A, Shameem M, Husain Q. Altered oxidant-antioxidant levels in the disease prognosis of chronic obstructive pulmonary disease. Int J Tuberc Lung Dis. 2013;17:1104-1109. 
22. Ruprai RK. Plasma oxidant-antioxidants status in asthma and its correlation with pulmonary function tests. Indian J Physiol Pharmacol. 2011;55:281-7.

23. Taborda-Barata L, Potter PC. Socio-epidemiological Aspects of Respiratory Allergic Diseases in Southern Africa. World Allergy Organ J. 2012;5:1-8.

24. Fukai T, Ushio-Fukai M. Superoxide dismutases: role in redox signaling, vascular function, and diseases. Antioxid Redox Signal 2011;15:1583-1606.

25. Dave L, Saxena T, Pathak S, Shrivastava N, Mathur AK. A study of oxidantsantioxidants balance in asthmatic patients. J Evol Med and Dent Sci/ elSSN- 2278-4802, pISSN-2278-4748/ Vol. 3/ Issue 23. 2014
26. Sree Yerrajwala K. Evaluation of antioxidants in patients with chronic obstructive pulmonary disease (COPD). Int J Med Sci Pub Health. 2016:5:1374-1379.

27. Yao X, Dai C, Fredriksson K, Lam J, Gao M, Keeran KJ, et al. Human Apo lipoprotein E genotypes differentially modify house dust miteinduced airway disease in mice. Am J Physiol Lung Cell Mol Physiol. 2012a;302:L206-L215

28. Yao X, Vitek MP, Remaley AT, Levine SJ. Apo lipoprotein mimetic peptides: a new approach for the treatment of asthma. Front Pharmacol. 2012b;3:37.

This work is licensed under a Creative Commons Attribution-NonCommercial 3.0 Unported License which allows users to read, copy, distribute and make derivative works for non-commercial purposes from the material, as long as the author of the original work is cited properly. 